\title{
Towards Eliciting Feedback for Artworks on Public Displays
}

Jonas Oppenlaender

University of Oulu

Oulu, Finland

jonas.oppenlaender@oulu.fi

\section{Simo Hosio}

University of Oulu

Oulu, Finland

simo.hosio@oulu.fi

\begin{abstract}
The internet and digital technologies have had a strong influence on how art is created, distributed and perceived. Museums and art galleries, however, are still predominantly places of passive consumption of art. In our project, we explore new forms of communication and feedback between artists and their audience, mediated by public displays. In this work-in-progress paper, we present a situated feedback system for giving feedback on artworks in a public setting. We present a preliminary evaluation of the system with
\end{abstract}

Permission to make digital or hard copies of part or all of this work for personal or classroom use is granted without fee provided that copies are not made or distributed for profit or commercial advantage and that copies bear this notice and the full citation on the first page. Copyrights for third-party components of this work must be honored. For all other uses, contact the Owner/Author.

C\&C '19, June 23-26, 2019, San Diego, CA, USA

(C) 2019 Copyright is held by the owner/author(s).

ACM ISBN 978-1-4503-5917-7/19/06.

https://doi.org/10.1145/3325480.3326583 artists and potential audience on their reactions to the system and eight different types of feedback.

\section{Author Keywords}

Public Displays; Feedback; Situated Feedback; Digital

Art; Situated Crowdsourcing.

\section{CCS Concepts}

- Human-centered computing $\rightarrow$ Displays and imagers;

- Human-centered computing $\rightarrow$ Graphical user

interfaces; • Human-centered computing $\rightarrow$ User

studies; • Information systems $\rightarrow$ Crowdsourcing

\section{Introduction}

The Internet and World Wide Web have opened an opportunity for artists to seek feedback in online communities and on websites. Feedback on these websites is, however, unstructured and often of low quality [1]. Researchers have consequently investigated ways of improving the feedback with better structure [4], rubrics [7], and scaffolding [8]. Online Feedback Exchange systems [2] and crowd feedback systems $[3,4,5,6]$ are computer-mediated systems that enable individuals to collect feedback from a large and diverse number of people.

Art communicates the social and personal values of the artist [13]. Given this cognitive and social function of art, communication and feedback between the audience and the artist are an underexplored research area. 
Due to the growing synergies between art and technology and the affordances of locative media [9], public displays can be used to exhibit digital art [10] and elicit situated interactive feedback $[11,12]$.

\section{Feedback on Public Displays}

Public displays have several unique characteristics that make them ideal for serendipitously collecting feedback via situated crowdsourcing [21]. Public displays attract the attention of passersby. This characteristic is well researched and has been exploited in numerous public display installations all over the world $[18,19,20]$. A further advantage of public display feedback systems is that they allow for collecting feedback from uncompensated samples of the audience. With such a system, an artist may tap into a large pool of people at no cost to receive authentic and rich contextual feedback. Public displays further allow for recording of nonverbal reactions of members of the audience.

While the feasibility of eliciting feedback has been proven in the literature, e.g. by Hosio et al. [11, 12], Goncalves et al. [16] reported that feedback on public displays may be of low quality and noisy, due to the often playful approach that users take in their interaction with this feedback medium. Consistent with the literature, we expect that giving the feedback a structure and using rubrics will significantly improve the quality of text-based feedback entered on public displays and the usefulness of the feedback for its receiver. Given that entering text on public displays may be perceived as cumbersome, we further hypothesize that public displays are especially suitable for collecting non-text based and visual feedback.

In this paper, we present a public display feedback system in the form of a digital art installation. We discuss the results of a preliminary evaluation of the system from two perspectives: artists and potential audience. First, we interviewed artists and inquired what feedback they would like to receive on public displays in general. We then presented them with eight pre-defined feedback mechanisms. Second, we evaluated the eight different feedback mechanisms with potential members of the audience.

\section{Digital Art Installation for Eliciting Situated}

\section{Feedback from the Audience}

In this section, we present the DUPLEX concept of an interactive digital artwork installation allowing for bidirectional communication to take place between artists and audience using public displays (see Figure 1). The concept comprises a large public display (main display) depicting one digital artwork at a time. A touchscreen input device is placed in front of the main display. Both the main display and the input device are always awake to indicate the affordance of interactivity to passersby and to combat interaction blindness [14].

Artists from anywhere in the world can upload their digital artworks to this digital art installation by visiting a dedicated web page with an upload form. Uploaded artworks are entered in a queue after review by a researcher to ensure that unsuitable work is not displayed in the public installation. The fundamental difference to a traditional gallery setting, as found in museums and art galleries, is the interactivity of the installation that allows for communication to take place between the audience and the artist, mediated by the public display. 


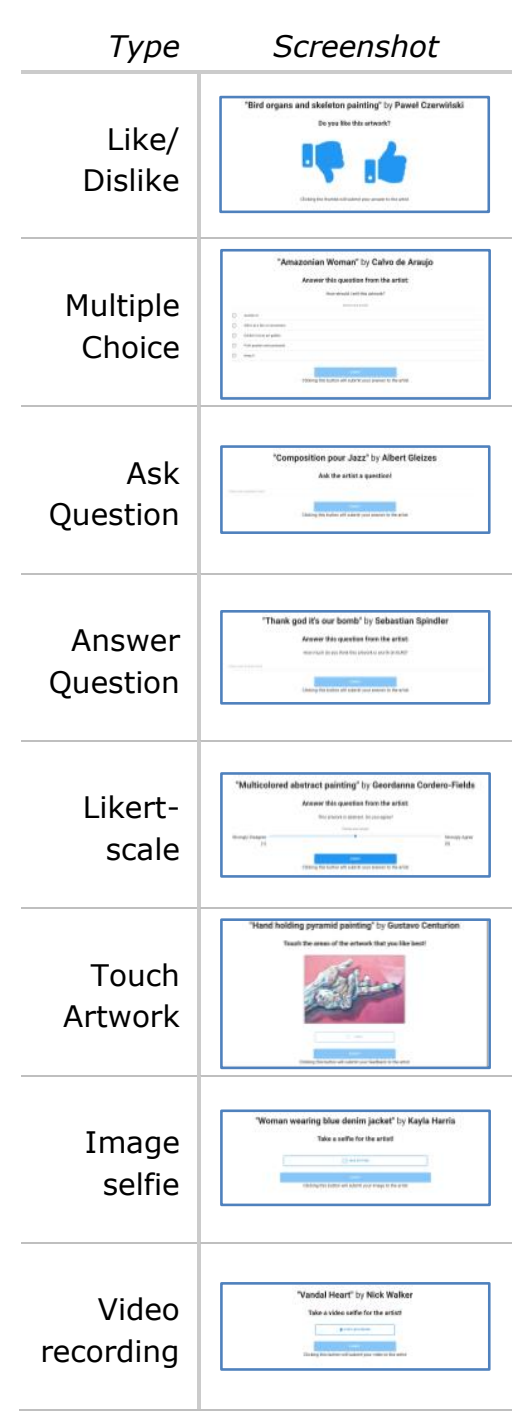

Table 1: Pre-defined feedback mechanisms in our studies.

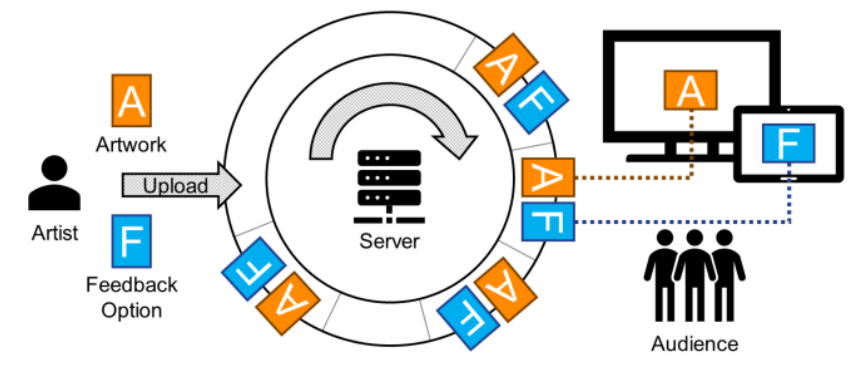

Figure 1: Illustration of the DUPLEX concept.

Communication from Audience to Artist

The audience controls the art installation by giving feedback on the touchscreen device. Once feedback is given for an artwork, a new artwork is displayed on the main display and feedback is elicited for this artwork. The two-screen setup was chosen because it avoids issues relating to showing personal content on public screens [17]. By giving the member of the audience a private screen for data entry, we avoid these privacyrelated issues. Bystanders can still appreciate the artwork on the large display while feedback is entered privately on the small display.

\section{Communication from Artist to Audience}

Artists will be able to choose from a list of pre-defined feedback options, or freely define their own feedback. Artists could exploit the art installation in unexpected ways by giving the audience a task to complete. Our system could, for instance, be used to deliver interactive experiences to the audience. Our system allows for recording of non-verbal reactions and even staged responses, enactments and performative interactions [5, 23], potentially complementing the artwork and its viewing experience. Our installation could also be appropriated to conduct experiments with the situated crowd, for example to explore potentially surprising interactions.

Allowing for Appropriation of the System Appropriation is the adaption of technology by its users in ways the designer did not envisage [22]. We encourage appropriation of the installation by the artist and by the audience. In doing so, we are interested in what kind of communication and strategies between artists and audience will emerge and how the audience reacts to the possibly surprising interaction with the artist.

\section{Pedagogical Purpose of the Installation}

We intend to bootstrap the art installation with artworks from young art students from local art schools. The system could therefore provide authentic feedback to the new generation of aspiring artists.

\section{Implementation}

We implemented the feedback system with a lightweight prototype, depicted in Figure 2 . The system consists of a display and an Android tablet on a lightweight floor stand. The mobile application running on the tablet was developed with Apache Cordova. The display is connected to a laptop computer which displays a maximized browser window. Both the tablet and the computer are connected to a Web server via WebSocket connections. The WebSocket server pushes the artwork to the main display and the associated feedback to the mobile application on the tablet. Feedback is stored in a MongoDB database on the server. Images and videos are also stored locally on the tablet and are automatically backed-up to Google Photos in the cloud.

We implemented eight atomic feedback mechanisms (see Table 1 in the Sidebar and the example in Figure 3). 


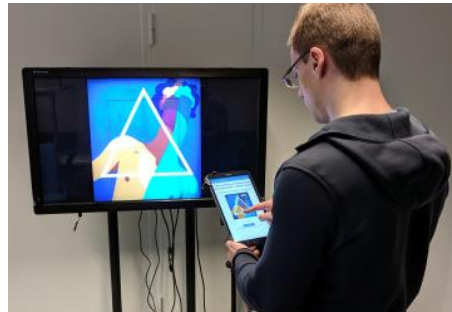

Figure 2: A person using the prototype in the laboratory study. Artwork credit: Stéphan Valentin.

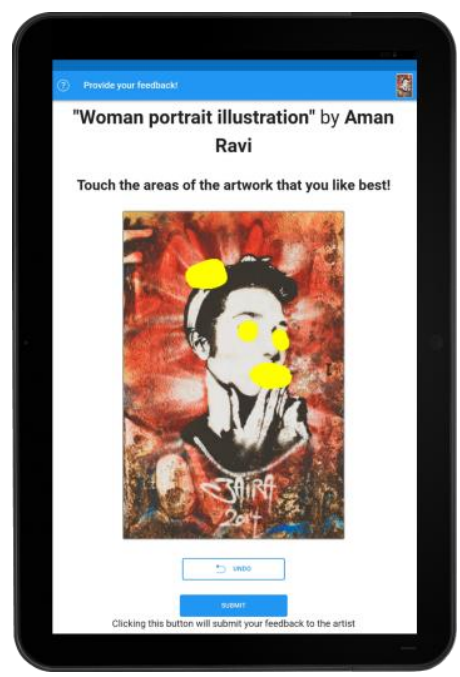

Figure 3: An example of feedback provided with the "Touch Artwork" feedback. Artwork credit: Aman Ravi.
These feedback mechanisms were selected because they represent some of the most common forms of data entry in online questionnaires. We hand-picked eight different artworks from a list of public domain artworks on Wikimedia Commons and from Unsplash, a stock photography website. We selected the artworks based on two criteria. First, the artworks are modern and appear to be created by contemporary artists. For instance, the collection contains several depictions of street art. Second, we selected artworks from different styles. The collection contains some very abstract pieces of art, but also some more realistic ones.

\section{Needfinding Study with Artists}

We invited two young local artists (A1, age 26, and A2, age 23, each with around three years of experience as an active artist) to an hour-long interview session. The artists were each compensated with a cinema ticket and coffee voucher worth US $\$ 15$. We first presented the prototype to the artists without going into detail about the different feedback types. Our aim was to find out the first impressions of the installation on the artists, how the installation would benefit them, and what kind of feedback the artists would find useful. We then let each of the artists use the installation while thinking aloud. The discussion unfolded around the needs of the artists and their requirements for receiving feedback.

First impressions were positive, with the installation being of immediate use to both artists. The artists' thought that the installation would solve three important needs: 1) developing one's skills, 2) receiving confirmation from the crowd, and 3) satisfying the need to market one's artworks and person. We briefly report on each of these three aspects.

\section{Skill Development}

The public display installation could be used to advance one's artistic skills. The feedback system would allow to "identify both one's weaknesses and strengths" and to expand one's skill set by complementing or contrasting existing skills (A2). Both artists agreed that they would not be willing to publish their unfinished artworks on public displays. This finding confirmed our initial assumption that the public display installation would be best suitable for showcasing finished artworks, as opposed to unfinished art and draft designs.

\section{Confirmation from the Crowd}

The feedback system could provide confirmation to artists. First, an artist could validate if the intention and meaning of an artwork is understood by the audience. Both artists regarded this as valuable information. Second, this type of feedback could provide the artist with positive self-affirmation (A1).

\section{Marketing Purposes}

Besides the immediate benefit of exhibiting artworks on public displays, as documented by Kukka et al. [10], the art installation was regarded as useful for establishing a brand and positioning oneself as an artist. A1 argued that with the help of the installation, artists could prove their reputation to potential buyers and gallerists. The system could be used to establish and validate a reputation in a certain niche.

Value of the Feedback Types for the Artists

Based on our observation in the workshop, the usefulness of the eight different feedback types for the artists is listed in Table 2. In this table, one star represents a low usefulness of the feedback for the artists, and three stars the highest usefulness. 


\section{User Study}

Following our needfinding study with artists, we were also interested in exploring how the audience perceives the installation and the different feedback mechanisms. To this end, we conducted a user study with 12 participants (aged 21 to 52 , mean age 28, 5 females). The participants were recruited on our campus with posters and e-mail lists.

The participants were invited to use our feedback system in a laboratory setting under the observation of a researcher. Each participant was given a short briefing about the purpose of the study and the installation, and then provided feedback for each of the eight pre-defined feedback types. Both the order of the artworks and the associated feedback types were randomized after each trial.

\begin{tabular}{|c|c|c|c|}
\hline Feedback & Artists & Viewpoint & Audience $^{1}$ \\
\hline Like/Dislike & * & & $3.58(1.38)$ \\
\hline Likert-scale & $* * *$ & $=1$ & $4.17(0.94)$ \\
\hline Multiple Choice & $* * *$ & & $3.92(0.90)$ \\
\hline Answer Question & ** & & $3.42(1.08)$ \\
\hline Ask Question & * & $\mid<$ & $3.67(1.30)$ \\
\hline Touch Artwork & $* * *$ & $=$ & $4.17(1.03)$ \\
\hline Image selfie & * & $=$ & $2.33(1.55)$ \\
\hline Video recording & ** & $>1$ & $2.92(1.24)$ \\
\hline
\end{tabular}

Table 2: Viewpoints of artists and audience. Opposing views are marked in blue, agreement in green.

1 Mean responses (and standard deviation) for the question "Overall, how do you like this feedback", on a scale from 1 (Strongly Dislike) to 5 (Strongly Like).
Immediately following the trial, we collected information with an online questionnaire and unstructured interviews. Participation took around 20 minutes and was compensated with a coffee voucher worth US $\$ 5$.

Results

The mean responses and standard deviation are listed in Table 2. Touching and painting areas of the artwork was engaging the participants. Likert-scale and multiple choice were perceived as an easy way of giving feedback. Selfie images were a surprising interaction. 8 out of the 12 participants reported that they did not know what to do. Unexpectedly, three participants used the video feedback to verbally give feedback to the artist.

\section{Discussion and Future Work}

With this situated system, we aim to not only elicit feedback from a diverse audience with different perspectives and experiences, but also to "configure spaces for imagination and dialogue that make those differences sensible as well as acceptable and valuable" [23] to two groups of actors: artists and their audience.

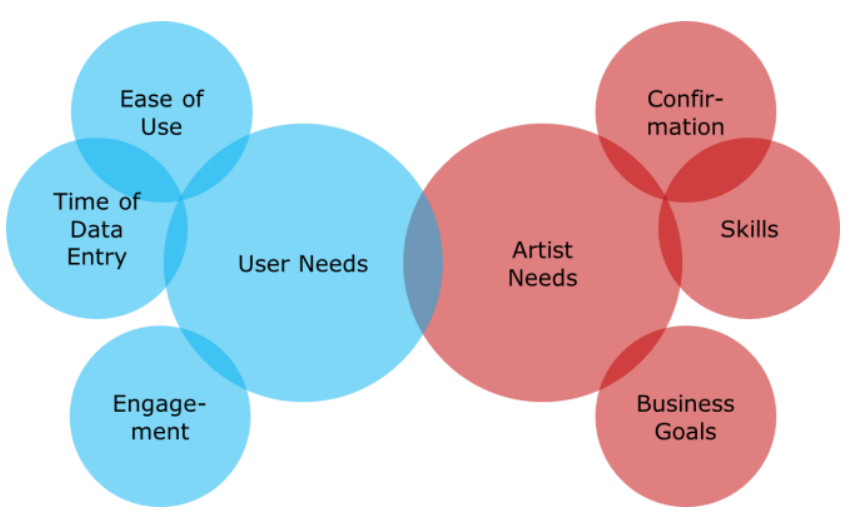

Figure 4: User needs and artist needs. 
The artists and participants in our study concur in their assessment of several types of feedback, but disagree in others (see Table 2 and Figure 4). Since "dissensus is the engine of creativity" [23], we are interested in exploring these opposing viewpoints in future work. Selfie images, for instance, can be highly engaging for certain members of the audience [15]. The two artists in our needfinding study, however, deemed selfie images as not valuable. Further, while the artists regarded seeing videos of reactions to their artwork as useful, the audience was confused by this surprising interaction. Lastly, the participants liked the convenience of giving feedback in the form of a simple thumbs up/down rating, while artists disliked the low informational value of this feedback type. Future research will need to contrast and reconcile such differences to find a common ground.

As a first inquiry, our study is system-driven, rather than user-driven. In future work, we envision that artists could appropriate the feedback system for their own purposes. We see the atomic feedback types from our study as components that could be used to compose more complex feedback mechanisms. For instance, artists could combine visual feedback that allows the audience to draw on the artwork (see Figure 3) with free-form text feedback to inquire "which parts do you dislike and why." Such compositions of tasks and atomic feedback types have the potential to provide a benefit beyond the mere text-based feedback of online communities and many crowd feedback systems.

\section{Conclusion}

We presented a situated feedback system for digital art on public displays. In a needfinding and user study, we investigated the different needs of two main types of users of this public display feedback system: artists and potential members of the audience. The artists in our study were positively impressed and excited about the opportunities that our feedback system could potentially afford them. Our user study established the feasibility of the feedback system and furthered our understanding of which different atomic feedback mechanisms may be most suitable for eliciting feedback on a public display installation. Overall, our studies constitute evidence of the potential of public display installations for eliciting summative feedback from the situated crowd.

With our art installation with situated feedback on public displays, we seek to stimulate communication between artist and audience and to establish new forms of audience participation on public displays. We aim to contribute towards a greater understanding of how public displays may allow the reimagination of traditionally co-located feedback interactions.

\section{Acknowledgements}

The first author gratefully acknowledges the SIGCHI student travel grant.

\section{References}

[1] Anbang Xu and Brian Bailey. 2012. What Do You Think?: A Case Study of Benefit, Expectation, and Interaction in a Large Online Critique Community. In Proceedings of the ACM 2012 Conference on Computer Supported Cooperative Work (CSCW '12). ACM, New York, NY, USA, 295-304. DOI: https://doi.org/10.1145/2145204.2145252

[2] Eureka Foong, Steven P. Dow, Brian P. Bailey, and Elizabeth M. Gerber. 2017. Online Feedback Exchange: A Framework for Understanding the SocioPsychological Factors. In Proceedings of the 2017 CHI Conference on Human Factors in Computing Systems 
(CHI '17). ACM, New York, NY, USA, 4454-4467. DOI: https://doi.org/10.1145/3025453.3025791

[3] Steven Dow, Elizabeth Gerber, and Audris Wong. 2013. A Pilot Study of Using Crowds in the Classroom. In Proceedings of the SIGCHI Conference on Human Factors in Computing Systems (CHI '13). ACM, New York, NY, USA, 227-236. DOI:

https://doi.org/10.1145/2470654.2470686

[4] Kurt Luther, Jari-Lee Tolentino, Wei Wu, Amy Pavel, Brian P. Bailey, Maneesh Agrawala, Björn Hartmann and Steven P. Dow. 2015. Structuring, Aggregating, and Evaluating Crowdsourced Design Critique. In Proceedings of the 18th ACM Conference on Computer Supported Cooperative Work \& Social Computing (CSCW '15). ACM, New York, NY, USA, 473-485. DOI:

https://doi.org/10.1145/2675133.2675283

[5] Judith Redi and Isabel Povoa. 2014. Crowdsourcing for Rating Image Aesthetic Appeal: Better a Paid or a Volunteer Crowd? In Proceedings of the 2014 International ACM Workshop on Crowdsourcing for Multimedia (CrowdMM '14). ACM, New York, NY, USA, 25-30. DOI:

https://doi.org/10.1145/2660114.2660118

[6] Anbang Xu, Shih-Wen Huang, and Brian Bailey. 2014 Voyant: Generating Structured Feedback on Visual Designs using a Crowd of Non-Experts. In

Proceedings of the 17th ACM conference on Computer supported cooperative work \& social computing (CSCW '14). ACM, New York, NY, USA, 1433-1444. DOI: https://doi.org/10.1145/2531602.2531604

[7] Alvin Yuan, Kurt Luther, Markus Krause, Sophie Isabel Vennix, Steven P Dow, and Bjorn Hartmann. 2016. Almost an Expert: The Effects of Rubrics and Expertise on Perceived Value of Crowdsourced Design Critiques. In Proceedings of the 19th ACM Conference on Computer-Supported Cooperative Work \& Social Computing (CSCW '16). ACM, New York, NY, USA, 1005-1017. DOI:

https://doi.org/10.1145/2818048.2819953

[8] Michael D. Greenberg, Matthew W. Easterday, and Elizabeth M. Gerber. 2015. Critiki: A Scaffolded
Approach to Gathering Design Feedback from Paid Crowdworkers. In Proceedings of the 2015 ACM SIGCHI Conference on Creativity and Cognition (C\&C '15). ACM, New York, NY, USA, 235-244. DOI: https://doi.org/10.1145/2757226.2757249

[9] Marc Tuters and Kazys Varnelis. 2006. Beyond Locative Media: Giving Shape to the Internet of Things. Leonardo 39, 4, 357-363.

[10] Hannu Kukka, Johanna Ylipulli, Jorge Goncalves, Timo Ojala, Matias Kukka, and Mirja Syrjälä. 2017. Creator-centric Study of Digital Art Exhibitions on Interactive Public Displays. In Proceedings of the 16th International Conference on Mobile and Ubiquitous Multimedia (MUM '17). ACM, New York, NY, USA, 3748. DOI: https://doi.org/10.1145/3152832.3152835

[11] Simo Hosio, Jorge Goncalves, Vassilis Kostakos, and Jukka Riekki. 2015. Crowdsourcing Public Opinion Using Urban Pervasive Technologies: Lessons From Real-Life Experiments in Oulu. Policy \& Internet, 7, 2 (2015), 203-222.

[12] Simo Hosio, Jorge Goncalves, Hannu Kukka, Alan Chamberlain, and Alessio Malizia. 2014. What's in it for me: Exploring the Real-World Value Proposition of Pervasive Displays. In Proceedings of The International Symposium on Pervasive Displays (PerDis '14), Sven Gehring (Ed.). ACM, New York, NY, USA, Article 174, 6 pages. DOI: https://doi.org/10.1145/2611009.2611012

[13] Howard Risatti. 1987. Art Criticism in Discipline-Based Art Education. Journal of Aesthetic Education 21, 2 (1987), 217-225.

[14] Simo Hosio, Hannu Kukka, Jorge Goncalves, Vassilis Kostakos, and Timo Ojala. 2016. Toward Meaningful Engagement with Pervasive Displays. IEEE Pervasive Computing15, 3 (July 2016), 24-31. DOI: https://doi.org/10.1109/MPRV.2016.58

[15] Simo Hosio, Richard Harper, Kenton O'Hara, Jorge Goncalves, and Vassilis Kostakos. 2015. Life Through the Lens: A Qualitative Investigation of Human Behaviour with an Urban Photography Service. In Proceedings of the 2015 British HCI Conference 
(British HCI '15). ACM, New York, NY, USA, 157-164. DOI: http://dx.doi.org/10.1145/2783446.2783577

[16] Jorge Goncalves, Simo Hosio, Yong Liu, and Vassilis Kostakos. 2014. Eliciting Situated Feedback: A Comparison of Paper, Web Forms and Public Displays. Displays 35, 1 (2014), 27-37. DOI:

https://doi.org/10.1016/j.displa.2013.12.002

[17] Dominik Weber, Alexandra Voit, Gisela Kollotzek, Lucas van der Vekens, Marcus Hepting, Florian Alt, and Niels Henze. 2018. PD Notify: Investigating Personal Content on Public Displays. In Extended Abstracts of the $2018 \mathrm{CHI}$ Conference on Human Factors in Computing Systems (CHI EA '18). ACM New York, NY, USA, Paper LBW014, 6 pages. DOI: https://doi.org/10.1145/3170427.3188475

[18] Oliver Storz, Adrian Friday, Nigel Davies, Joe Finney, Corina Sas, and Jennifer Sheridan. 2006. Public Ubiquitous Computing Systems: Lessons from the eCampus Display Deployments. IEEE Pervasive Computing 5, 3 (July 2006), 40-47. DOI: https://doi.org/10.1109/MPRV.2006.56

[19] Timo Ojala, Vassilis Kostakos, Hannu Kukka, Tommi Heikkinen, Tomas Linden, Marko Jurmu, Simo Hosio, Fabio Kruger, and Daniele Zanni. 2012. Multipurpose Interactive Public Displays in the Wild: Three Years
Later. Computer 45, 5, 42-49. DOI:

http://dx.doi.org/10.1109/MC.2012.115

[20] Tommi Heikkinen, Tomas Linden, Timo Ojala, Hannu Kukka, Marko Jurmu and Simo Hosio. 2010. Lessons Learned from the Deployment and Maintenance of UBI-Hotspots. Proceedings of the 4th International Conference on Multimedia and Ubiquitous Engineering. IEEE, Cebu, Philippines, 1-6. DOI https://doi.org/10.1109/MUE.2010.5575054

[21] Jorge Goncalves, Denzil Ferreira, Simo Hosio, Yong Liu, Jakob Rogstadius, Hannu Kukka, and Vassilis Kostakos. 2013. Crowdsourcing on the Spot: Altruistic Use of Public Displays, Feasibility, Performance, and Behaviours. In Proceedings of the 2013 ACM international joint conference on Pervasive and ubiquitous computing (UbiComp '13). ACM, New York NY, USA, 753-762. DOI: https://doi.org/10.1145/2493432.2493481

[22] Alan Dix. 2007. Designing for Appropriation. In Proc. British HCI Group Annual Conf. People and Computers (BCS-HCI '07), Vol. 2. BCS Learning \& Development Ltd., Swindon, UK, 27-30.

[23] Peter Wright and John McCarthy. 2015. The Politics and Aesthetics of Participatory HCI. Interactions 22, 6 (October 2015), 26-31. DOI:

https://doi.org/10.1145/2828428 